\title{
A Visualization System for Exploring Logo Trend and Design Shape Patterns
}

\author{
Hyoji Ha ${ }^{1}$, Hyunwoo Han ${ }^{1}$ and Kyungwon Lee ${ }^{2, *}$ \\ 1 Lifemedia Interdisciplinary Program, Ajou University, Suwon 16499, Korea; hjha0508@ajou.ac.kr (H.H.); \\ ainatsumi@ajou.ac.kr (H.H.) \\ 2 Department of Digital Media, Ajou University, Suwon 16499, Korea \\ * Correspondence: kwlee@ajou.ac.kr
}

Received: 26 May 2020; Accepted: 29 June 2020; Published: 1 July 2020

\begin{abstract}
A logo is an effective way of expressing a brand's identity and an essential element in conveying the values and image of the company. The development process of a competitive logo should be based on a design that is future-proof in a rapidly changing global market; hence, understanding the design trends for successful logo design is key. In this study, the design shape elements of logo trend models were analyzed and made into a database. Then, a trend analysis system was produced using radial visualization (RadViz) and circular parallel coordinates data visualization techniques. RadViz allows observation of clusters of logos that have similar shape elements, whereas with circular parallel coordinates plots, detailed information of the shape elements of each logo trend can be seen. Using the system, it was confirmed that shape elements-such as transformation to surface, overlapping, artificiality, concept of color and rhythm - play a major role in driving a trend. It was observed that trends change over time as various shape elements are added or removed. In addition, our study is expected to help predict the logo trend models that will come into style in the future. While similar efforts have been made in the past, our proposed system improves upon them by utilizing standard design elements as the categorizing criteria, using a unique combination of RadViz and circular parallel coordinates data visualization techniques. Using our system as a guideline, many users would be able to create logos that reflect what is trending.
\end{abstract}

Keywords: logo trend; service design; data visualization; trend forecasting; design shape element

\section{Introduction}

\subsection{Research Background}

The modern design industry has become a competitive field in which artwork is produced not only for aesthetics but it also reflects individual preferences and conveys social and cultural values. In order to create a design that can satisfy various stakeholders, it is important that the design reflect the most popular trend. This has increased the need for understanding design trends [1].

A design trend is a socio-cultural phenomenon that reflects the dominant new idea of the times and has a great influence on design [2]. Therefore, it can provide a foundation for future design that can be a strong competitive force at the individual, corporate, or national level.

Many companies are now trying to choose a logo that represents the image of the company. The reason is that once the logo is chosen, it affects all aspects of the company such as image design, product design, and promotional material design [3]. Since the purpose of the logo is to catch people's attention, it has become very important to design symbols that follow the trend in order to compete with numerous other companies in a rapidly changing society. 
Today, the logos produced all over the world often reflect the popular social phenomena of the era $[4,5]$. For example, as shown in the top row of Figure 1, logos inspired by smartphone app icons appeared as a trend in the early 2010s, reflecting the fact that many people were using smartphone apps owing to the spread of smartphones. As shown in the bottom row of Figure 1, in 2018, there was a trend in which retro-styled fashion became popular again and illustrations inspired by hand-drawn images in the 1960s and 1970s were reinterpreted in the form of logos.
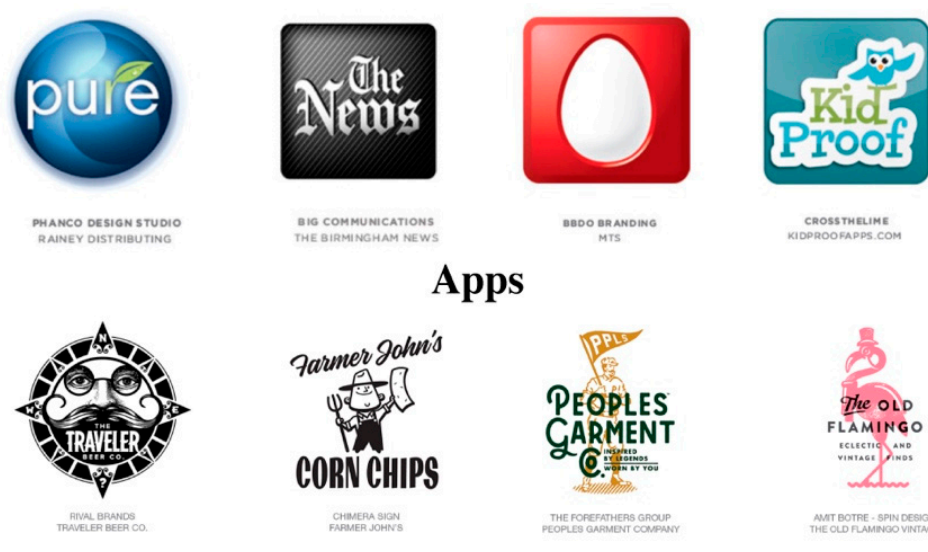

Apps
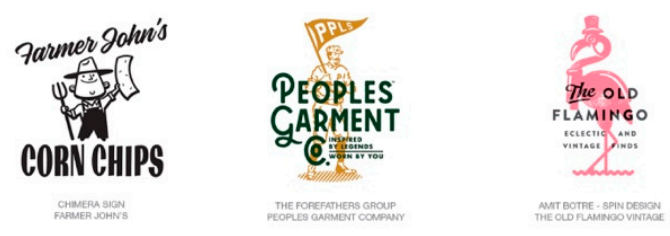

\section{Neo Vintage}

Figure 1. (Top) “Apps (2012)”, Trends Inspired by Smartphone App Icons, (Bottom) “Neo Vintage (2018)", Trends Inspired by the Retro Concept. Reproduced by using Reference [6]; 2020.

Therefore, in order for a logo to be noticed, it should incorporate a visual aesthetic that reads and interprets the trends of the era [7]. However, a good logo design cannot be created simply by following a trend unilaterally as it is most important that the logo meets the client's request or designer's intention [8,9]. Therefore, the selection of a trend requires more deliberation. Also, creating a single logo design requires a lot of research and effort so it is important to try out various trends for ideas. Thus, there is a definite need for more research on logo trends.

\subsection{Purpose of Research and Research Process}

The main purpose of this study is to classify the logo trend data according to design characteristics and then develop a logo trend visualization system based on the classified data. Our logo trend visualization system can identify the shape elements of a trend and identify the pattern of changes over time. We used the following research methods to achieve this purpose.

(1) Development of the design shape element classification system for logo trends and data generation: Using LogoLounge.com's annual Logo Trend Report (LogoLounge, Wichita, KS, USA) [4] as the data source, a total of $250 \operatorname{logo}$ trend articles were extracted from 2003 to 2019, and the design characteristics of the trends were analyzed. In this process, the criteria that a logo should meet are ascertained in order to classify them according to various trends.

(2) Development of the logo trend visualization system: The data collected through the classification system is used for developing the trend visualization system. The system consists of RadViz and circular parallel coordinates visualization methods. In RadViz, the clustering patterns of trends with similar shape elements can be seen. In circular parallel coordinates, detailed information of the shape elements of each logo trend can be seen.

(3) Case study to identify the trends of logos: The visualization system is used to identify the design trends of logos for each year, and the case study for forecasting logo trends that will be popular in the future is presented. In the case study, a target logo trend is set to explain how the logo trend transformed over time and what shape elements were added/removed in the process. The above research process is shown in Figure 2. 

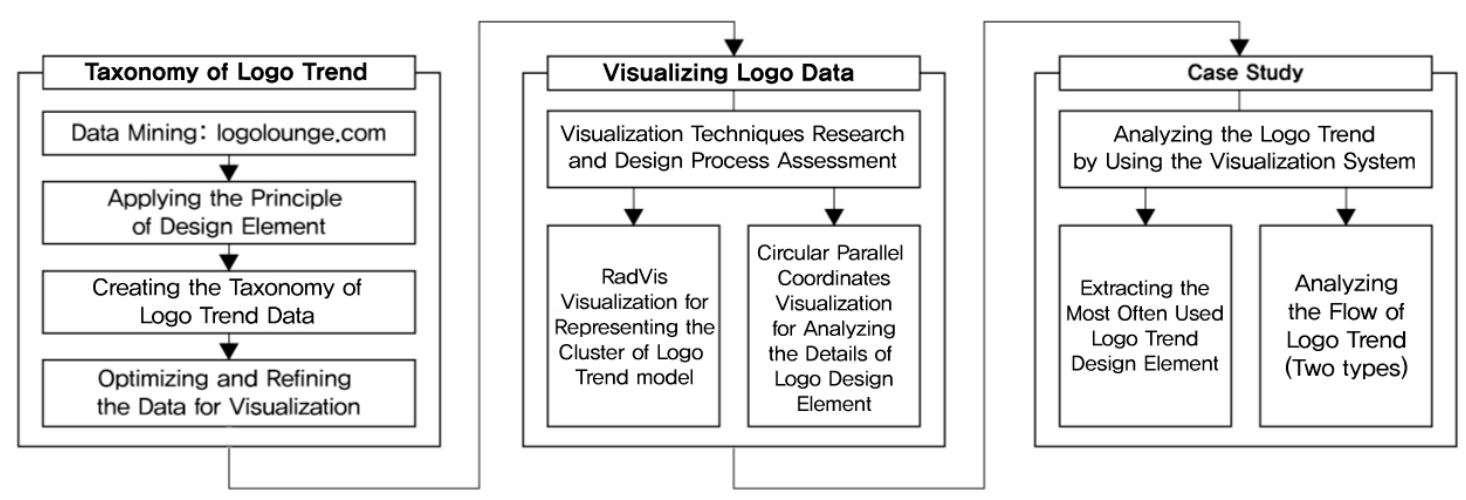

Figure 2. Research workflow.

The visualization system is used to identify the design trends of logos for each year, and the case study for forecasting logo trends that will be popular in the future is presented. This study was first introduced through a poster session at ACM VINCI2015 (8th The Symposium on Visual Information Communication and Interaction) [10] and we updated the visualization system by considering more diverse logo trend report data. In particular, the primary contributions of our study are briefly summarized here:

- A more detailed classification model of the design shape element (Table 1, Section 3) and the visualization development process (Section 5).

- An optimal visualization model has been established after implementing several visualization models that reveal the shape elements of the logo trend data and conducting assessments with experts.

- An analysis of eight clusters along with patterns of the design shape elements (Table 2) has been achieved using RadViz visualization.

- A report on the evolution of design shape element patterns from 2003 to 2019 has been created (Table 3).

- A case study is developed using abundant trend resources to discover the evolution process of specific logo trends. In this case study, we examined the following cases: (1) logo trends that evolved across different clusters in the RadViz visualization over time, and (2) logo trends that evolved over time within the same cluster pattern.

\section{Materials and Methods}

\subsection{Logo Classification}

While there have been previous attempts to classify logos, there have been no attempts made to classify logo trends [11]. The most representative example of logo classification is the Vienna classification [12], which is the standard for classification of graphical trademarks created by members of the Paris Convention to consistently classify the geometric elements included in trademarks. Each criterion is formed to define the geometric elements, consisting of 3 steps based on 29 categories, divisions, and sections.

In the Vienna classification, logos have been classified in terms of overall shape of icons (e.g., celestial shape, human shape, plant shape, letter shape, and furniture shape).

However, in order to classify this more systematically, it is necessary to use basic shape elements used in the design field as the criteria for the design element classification system of logos.

There are, of course, problems with the Vienna classification. Although there is a large category that utilizes the shape elements of design, it mostly consists of the logo cases designed in the mid-20th century, which is rather insufficient for studying the trend of logos. 
Therefore, for the cases focusing on the characteristics of the design pattern or the granular shape elements-such as "Concentrak, Selective Focus" in Figure 3-it is difficult to apply the method presented in the Vienna classification and a new classification system is thus required.

On the other hand, "LogoLounge.com" analyzes logos produced from around the world and suggests a trend by grouping them based on overall appearance or design pattern. This method of presentation can help in identifying trends for each year, but there are still limitations in predicting how the trends will evolve in the future.

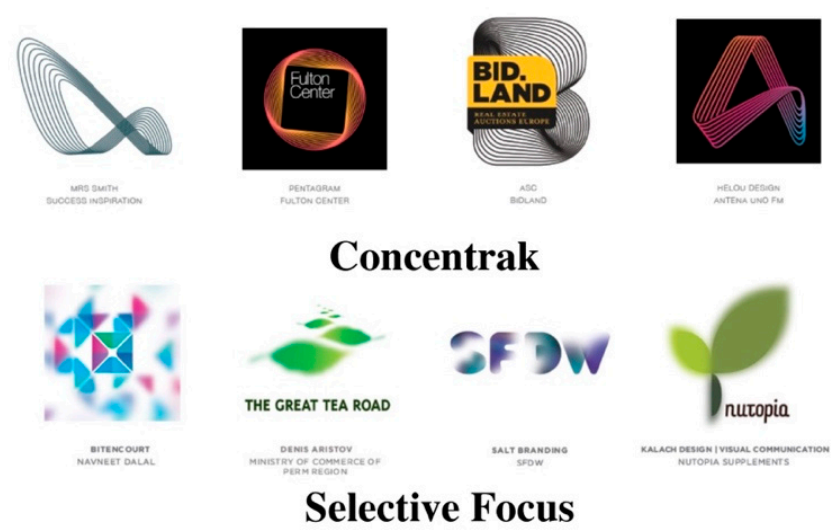

Figure 3. Examples of logo trends that are difficult to classify into concrete objects. Reproduced by using Reference [4]; 2020.

Therefore, this study intends to suggest not only the evolution of logo trends but also the prediction method for the trends that will become popular in the future with the help of logo trend visualization systems.

\subsection{Analysis of Logo Characteristics Using Engineering Technology}

There have been many studies around the world to determine the characteristics of logos based on image processing technology, and such techniques were used to search for similar logos or to determine the design aesthetics of brand logos. Figure 4 presents several works that proceeded the logo analysis using engineering technology.
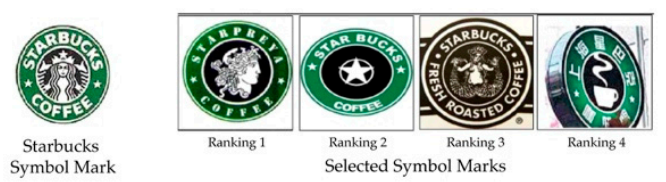

Kang et al. [13]

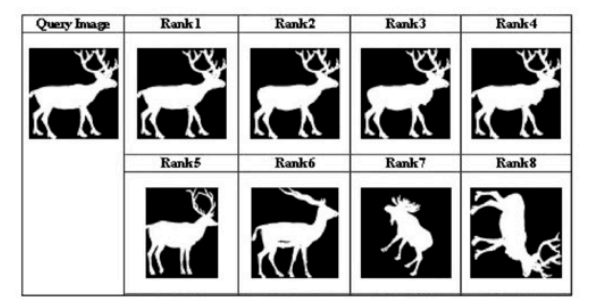

Qi et al. [15]

\begin{tabular}{|c|c|c|c|c|c|}
\hline $\begin{array}{l}\begin{array}{l}\text { Query } \\
\text { Image }\end{array} \\
\text { a }\end{array}$ & Rank 1 & Rank 2 & Rank 3 & Rank 4 & Rank 5 \\
\hline 2th & 㱍 & 业 & . & ats? & A \\
\hline int & $\leadsto$ & 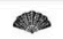 & तitin & $\Phi$ & 童全 \\
\hline
\end{tabular}

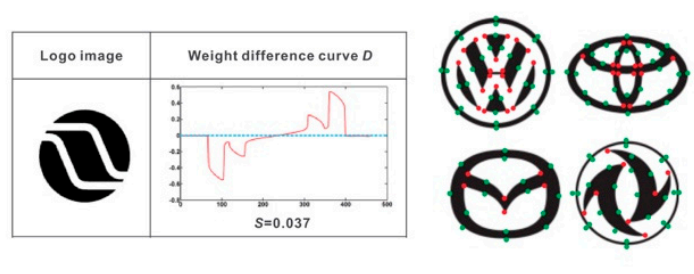

Zhang et al. [16]

Figure 4. Representative works of logo analysis using engineering technology. Reproduced from References [13-16]; 2010, 2009, 2010, 2017.

The study by Kang et al. [13] estimated the similarity of logos by calculating the numerical value for determining the geometric formation and detailed shape of a logo using image processing technology and then comparing similarity. As a result, logos similar to the logo of Starbucks were 
extracted. In the studies by Wei et al. [14] and Qi et al. [15], elements such as curvature, distance from the center point, etc., were quantified to determine the shapes appearing in the logos in order to determine similarity between various logos. However, the above studies have been carried out to measure the characteristics and shapes of logos and to classify the characteristics based on abstract representation, disregarding the basic information such as style, pattern, and characteristics of the transformations (distortion, repetition, and reflection) used by the shape elements. Hence, they are not suitable as a classification system that can be used easily and universally by many users.

In the study by Zhang et al. [16], the symbolic structures of famous brand companies with similar attributes were quantified and calculated by image processing. Then, the perceptual models of standard design principles were applied to evaluate each brand logo. In addition, the grades of balance, contrast, harmony, and aesthetics of logos were collected from a large number of volunteers. Finally, the collected grade data was used for machine learning to calculate the design patterns of the logos, which would be helpful in the process of determining the aesthetics of logos in the future. The above studies suggest the method to be used to determine aesthetics of logos and create trendy logos, using engineering technology. However, they do not suggest a serviced system for easy comprehension by logo makers or designers.

Therefore, in order to solve the above limitations, this study uses design shape elements that can be easily understood by those who develop logos. Moreover, in order to help logo designers come up with more competitive results, a visualization system has been presented to help them understand design element patterns in logos.

\subsection{Visualization}

Data visualization is used to improve readability by redesigning data analysis results. It has the advantage of providing insights that are not seen when data is presented simply in tables or graphs $[17,18]$. Among the various visualization methods currently available, multi-dimensional data visualization is used as a tool for clustering and arranging a large amount of data or a massive number of variables included in the data instance [19-24].

This study intends to produce trend visualizations using RadViz and circular parallel coordinates methods, two among various types of multi-dimensional data visualization techniques. The preliminary study data on the related visualization techniques are as follows. First, we take a look at the previous research related to RadViz [25-27]. RadViz is a method for visualization of variables in a dataset around a ring-shaped circle, where inside the circle, the data samples are represented as nodes [28]. According to Hoffman et al. [25], this visualization method arranges the data instance nodes in a two-dimensional map from n-dimensional points. The study by Pagliosa et al. [29] suggests that RadViz++ incorporate an icicle-plot metaphor into the existing RadViz visualization to show the clustering result of the data instance better. Zhou et al. [30] improved the layout of the RadViz visualization by using the mean shift algorithm. The algorithm finds the peaks of probability density in each dimension to transform the area of the dimensional anchor. This way, the nodes are clustered in the middle to some extent to resolve the overlap. However, it is difficult to figure out intuitively what values the data instances represented in RadViz have for each variable.

Parallel coordinates plots were first proposed by Inselberg [31-33] and are a common method for visualizing data values of multidimensional variables [34,35]. If there are $\mathrm{N}$ variables in the dataset, this method shows each variable in $\mathrm{N}$ axes with each axis aligned in parallel. The data instance included in the dataset is represented by the line graph with the numerical values for $\mathrm{N}$ variables. The study using parallel coordinates by Zhou et al. [36] proposed a clustering method using parallel coordinates curves and visual bundles. This method can observe the line graph of data instances represented in parallel coordinates from various viewpoints and has the advantage of being able to grasp the cluster pattern of data instances well. The study by Raseman et al. [37] proposed a system called "Parasol", using parallel coordinates visualization to enable an improved multi-objective decision-making experience for the users. It also provided functions to cluster, brush, link, and bundle data instances to reduce 
the clutter that occurs upon growth of data. However, the above studies have the problem of it being very difficult to follow the line graphs representing the data instances, as the spacing between the axes narrows with the increasing number of variables in the dataset in the existing layout that arranges the axes in parallel. In the study by Cheng et al. [38], parallel coordinates were used in addition to RadViz visualization to represent variable values of data instances. However, when presenting the two visualizations, the view was provided separately, which may result in gaze dispersion while the user interprets the data.

Our study intends to utilize both: the advantages of RadViz, which can easily organize the cluster relations between data instances, and the advantages of parallel coordinates, which intuitively displays the information of the variable values of data instances. Therefore, the parallel coordinates plot is displayed in the form of circles on the outside edge of the RadViz plots so that users can easily analyze the characteristics of the data instance [10].

To examine the characteristics of RadViz and parallel coordinates techniques thoroughly, this study applied the data of the logo design shape elements to both visualizations. Subsequently, we evaluated the data characteristics obtained from each visualization and derived pros and cons for each model. Furthermore, we conducted assessments with researchers having an experience of more than ten years in performing visualization techniques to receive feedback on such pros and cons. Details of these assessments have been covered in Section 4 .

\section{Design Shape Element Classification System for Logo Trends and Data Generation}

This study intended to extract and analyze a total of 255 logo trends from 2003 to 2019 using the annual Logo Trend Report of LogoLounge.com as a data source. Some logo trends have morphological characteristics, such as "Bubbles", "Potato chips", and "Buttons". Others are defined by colors, such as "Watercolor", "Green", and "Brown" [39]. There are also logos defined as layout and movement by multiple elements, such as "Overlays", "Icon clusters", and "Concentrak". In order to analyze such data, information on the most basic design principle in which a trend element of a logo can be generated should be reflected.

Therefore, in this study, the analysis criteria have been prepared by referring to the Principles of Form and Design [40], and the logo trends have been categorized by the design shape elements of logos. Principles of Form and Design by Wucius describes a collection of design shape elements that are the basis of design. This book also describes the various design principles for graphic producers and designers. It introduces the classification system mainly related to form/shape by describing basic definitions of points, lines, surfaces, and solids, definitions of complex design patterns, definitions of the relationships between forms (defining whether two forms are overlapping, touching, detached, etc.), and visual effects. The classification criteria of shape elements proposed in this study consist of concept element, visual element, correlation element, and the interrelationships of forms [40-42], and each basic element has several attributes (shown in Table 1).

This study analyzes the shape elements of each trend and quantifies the information using the logo shape elements in Table 1. The quantification task involved assessments that included two design experts with more than ten years of logo design experience, three graphic designers, and the authors of this study. The participants were given the images of the logo trend model one by one that were supplied by LogoLounge for comparison with the attributes provided in Table 1 and identification of the design shape for each trend. During this process, numerical values were calculated for the attributes that more than 70 percent of the participants agreed to be important. Especially, we increased the weights of attributes that the participants unanimously agreed to be important. These weights were reflected when we consider the nodes' position in the RadViz. The methods of calculating the weights and positioning the nodes are described in detail in Section 5.

Figure 5 shows an example of quantification of the logo trend characteristics (dataset download URL: https://bit.ly/3fwRILh). Column D in the figure is the trend name of the logo, and each trend is quantified according to criteria such as the concept element, visual element, correlation element, 
and the interrelationships of forms. Figure 6 is an example of analyzing each trend using the analysis criteria of Table 1. Figure 6 shows which design shape elements should be highlighted in order to form each logo trend.

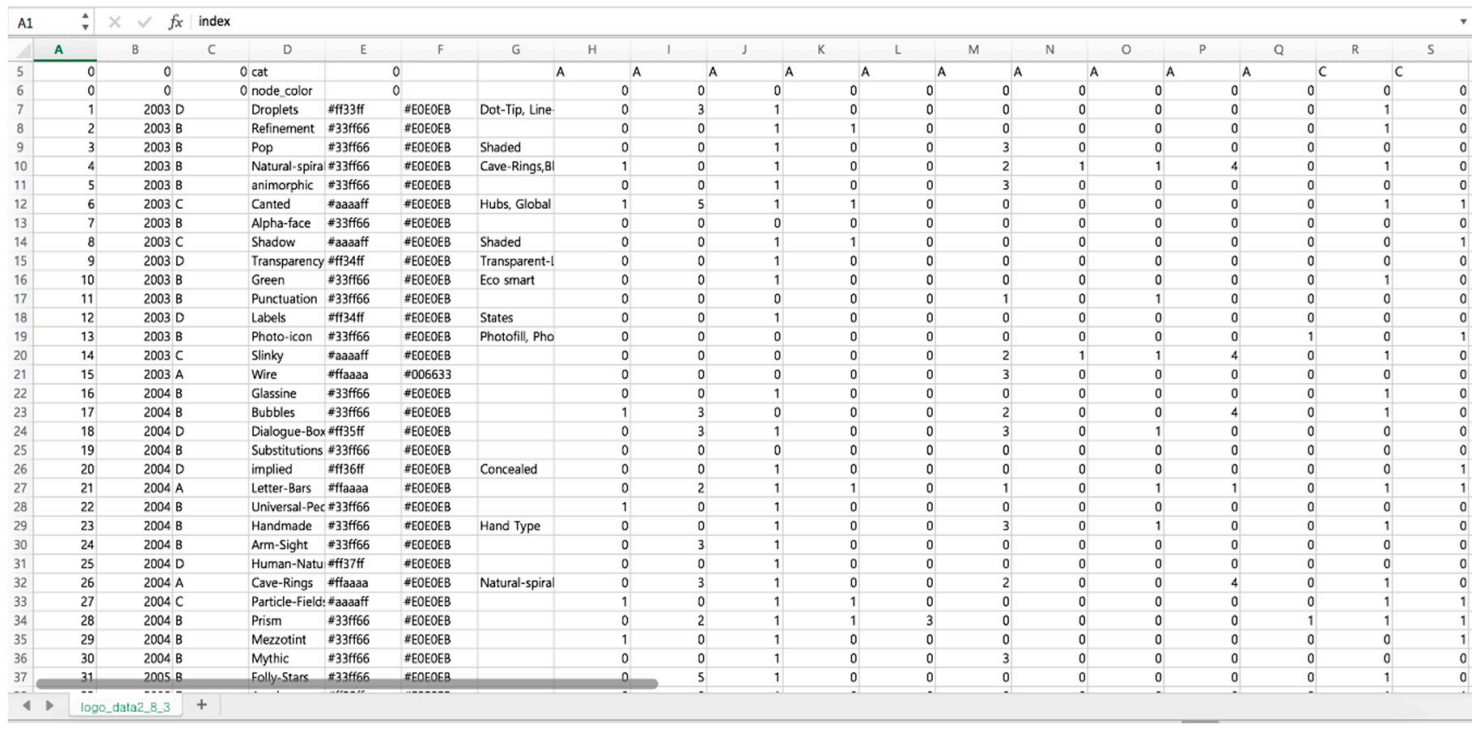

Figure 5. Quantification of logo trends based on design shape element criteria.

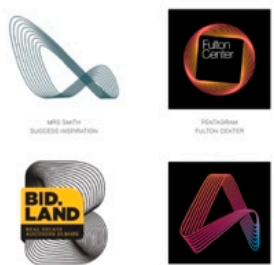

Concept element: Free curve, Line-Multidirectional, Transformation of surface Visual element: Geometry, Concept of color- Analogous, Texture

Correlation element: Rhythm, Space, Composition-Multi-direction

The interrelationships of forms: Detachment, Overlapping

\section{Concentrak}

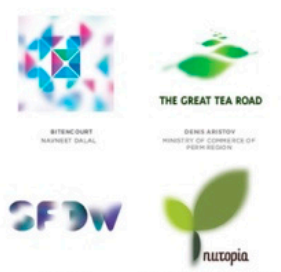

Concept element: Transformation of surface, Thickness, Speed

Visual element: Natural Object-Organism substance, Texture

Correlation element: Space

The interrelationships of forms: Union, Overlapping

\section{Selective Focus}

Figure 6. Example of logo trend analysis using shape element classification criteria for logos. 
Table 1. Design shape elements of logos.

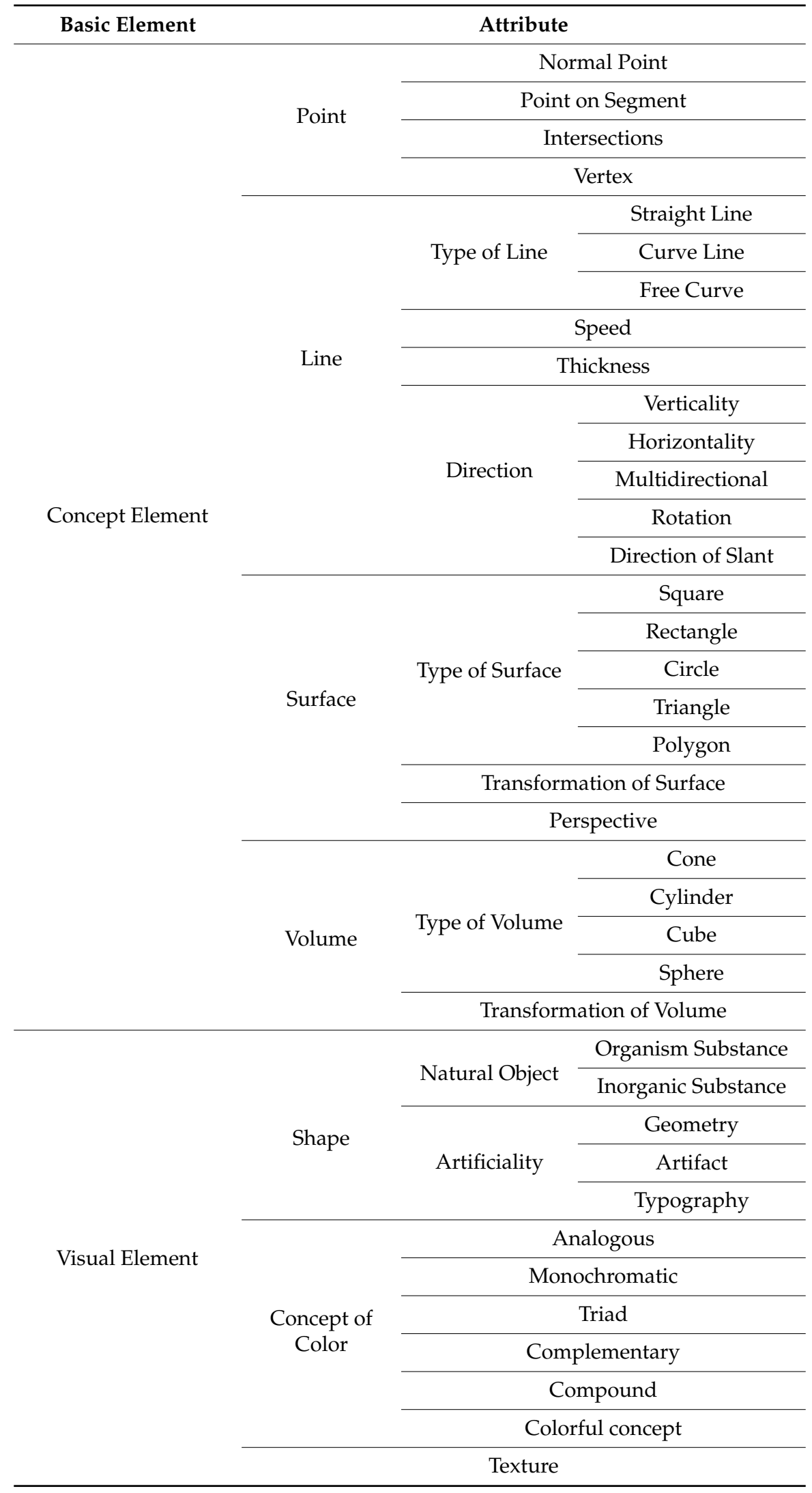


Table 1. Cont.

\begin{tabular}{|c|c|c|}
\hline Basic Element & & Attribute \\
\hline \multirow{6}{*}{ Correlation Element } & \multicolumn{2}{|r|}{ Rhythm } \\
\hline & \multicolumn{2}{|r|}{ Space } \\
\hline & \multicolumn{2}{|r|}{ Weight } \\
\hline & \multirow{3}{*}{ Composition } & One Direction \\
\hline & & Multi-direction \\
\hline & & Rotation \\
\hline \multirow{8}{*}{$\begin{array}{l}\text { The } \\
\text { Interrelationships of } \\
\text { Forms }\end{array}$} & \multicolumn{2}{|r|}{ Detachment } \\
\hline & \multicolumn{2}{|r|}{ Touching } \\
\hline & \multicolumn{2}{|r|}{ Overlapping } \\
\hline & \multicolumn{2}{|r|}{ Penetration } \\
\hline & \multicolumn{2}{|r|}{ Union } \\
\hline & \multicolumn{2}{|r|}{ Subtraction } \\
\hline & \multicolumn{2}{|r|}{ Intersection } \\
\hline & \multicolumn{2}{|r|}{ Coinciding } \\
\hline
\end{tabular}

\section{Design Process Assessment of Visualization}

This study has extracted the design shape elements to analyze the trends that significantly affect the logos. To determine the finest strategy to visualize the extracted data, we used RadViz and parallel coordinates.

As a brief introduction, the component principles of RadViz and parallel coordinates are mentioned again in Figure 7.

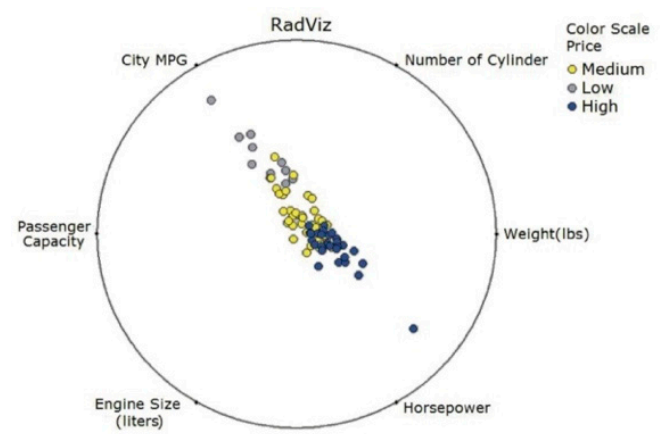

(a)

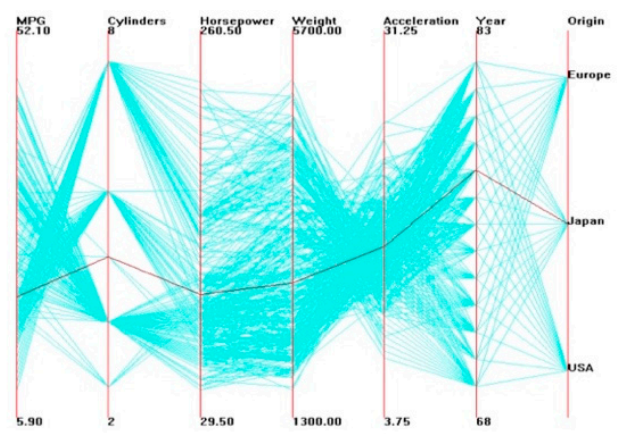

(b)

Figure 7. (a) RadViz, (b) parallel coordinates.

RadViz represents data attributes in the form of annular bands and data samples as nodes, where the nodes are located inside the circle loop. At the time, the nodes reflect position values of the attribute keywords included in the annular band according to the data attributes of the node [43]. These attribute keywords were called "dimensional anchors". The dimensional anchor refers to a point in the process where the applied strength of data instance nodes varies in accordance with their values $[44,45]$. Dimensional anchors are presented as points at equidistant intervals on a circumference. Figure 7a shows an example of a RadViz plot in which the color information of the node represents the price. The low-priced gray nodes are concentrated in the upper-left part of the circle due to efficiency (City MPG (miles per gallon): This variable was presented as the dimensional anchor in RadViz), while high-priced dark blue nodes are concentrated in the lower-right part due to horsepower and weight variables. 
A parallel coordinates visualization plot shows a set of points in N-dimensional space. It is generally in a vertical form and consists of $\mathrm{N}$ equally spaced parallel lines. It enables one to understand the relationship between variables in the data easily. According to Inselberg [31], parallel coordinates can be interpreted to have a positive correlation between two dimensions when most lines of each variable are parallel. When most lines intersect, they can be interpreted to have a negative correlation. In Figure $7 \mathrm{~b}$, each data instance is represented by a line graph. The relationship between the variable MPG and the variable cylinders is negative, while the relationship between the variable horsepower and the variable weight is positive, in general.

Based on these methods, we developed visualization models by using the quantification data of the logo trend characteristics, and we determined the pros and cons for each model. For this, we conducted a design process assessment based on visualization analysis. This process was based on qualitative feedback focusing on the opinions using the visualization technique.

First, we implemented logo trend visualization through the RadViz technique. The results are shown in Figure 8. Subsequently, we held an assessment to obtain the opinions of the experts on the pros and cons concerning the RadViz visualization results.

The visualization with RadViz showed the advantage of providing easily distinguishable logo trends with similar design shape elements clustered together in a mutual vicinity. In addition, some opinions indicated that the trends that were somewhat far apart from each other showed a distinctive difference in shape elements. Accordingly, the experts agreed that the shape flow of each trend could be identified by analyzing the logo characteristics constructed through RadViz (see the right side of Figure 8).
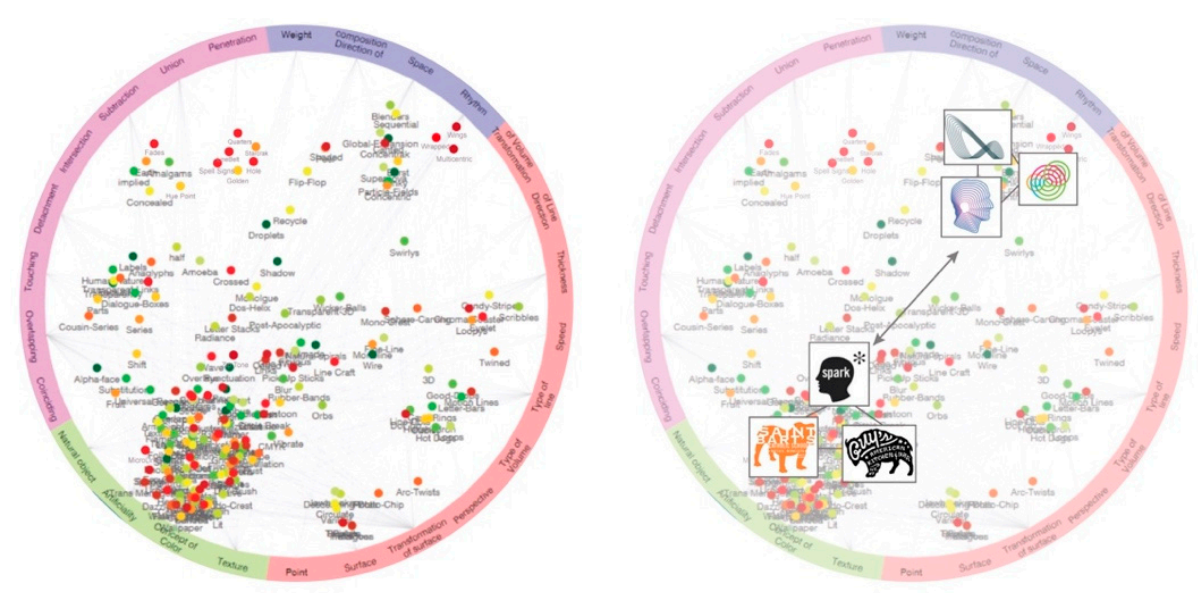

Figure 8. RadViz: A model for design process assessment.

In contrast, RadViz showed the disadvantage that it cannot identify the detailed design shape element information for each logo trend model. This implies that even if the logo trends are closely located, detailed information about whether the shape elements are similar or different cannot be obtained. Hence, in case of having only RadViz visualization, there was the inconvenience of identifying each logo model and evaluating the differences. Furthermore, it was pointed out that the size of the circles showing the visualization results should be increased to provide better readability because of the fact that too many variables define the shape elements. It was also pointed out that the users would face difficulties in understanding the high and low values of each design shape element variable for the logo trends located in the center of RadViz.

Second, based on the same logo data, we implemented the visualization of the logo trend using the parallel coordinates. Figure 9 shows the logo data visualization using such a technique. As shown in this figure, each logo is represented by a line, and the design shape variables that constitute the logo are constructed with each axis. We conducted an additional assessment on the visualization results. In general, parallel coordinates were convenient for carrying out a detailed analysis of the shape element information of the trend, given that it provides elements in order along the line graph of each 
axis. In addition, some opinions suggested that the parallel coordinates method has the advantage that it can filter and show only the logo trends that correspond to the shape element combinations desired by the designer.

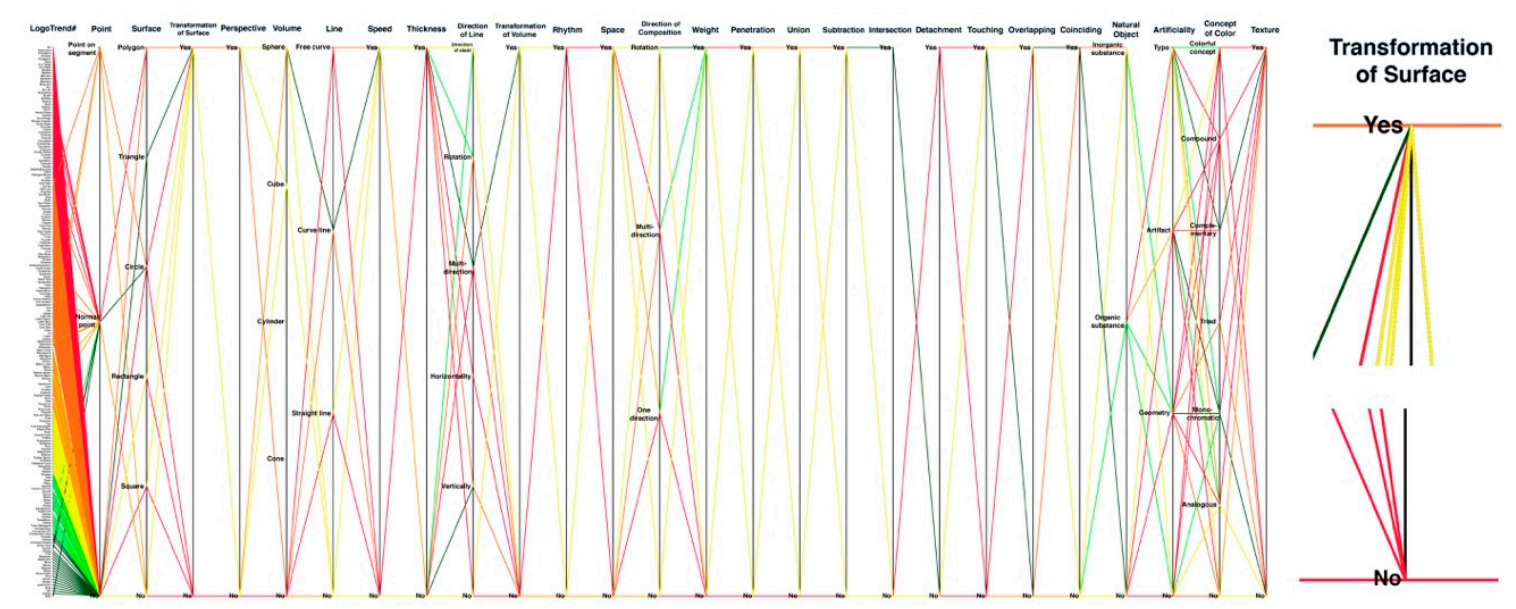

Figure 9. Parallel coordinates: another model for design process assessment.

However, as shown on the left side of Figure 9, it was pointed out that viewing the patterns of the entire line on the web may be difficult if the parallel coordinates layout having linear structure is applied. This is because of the large number of variables in the data and the close arrangement of the axis. Furthermore, given that many line graphs overlap, it has been indicated that quickly determining the number of logo trend models that are included at a certain point for each shape element may be difficult. More specifically, when looking at the right side of Figure 9, which is an enlarged portion of the parallel coordinates, numerous line graphs overlap in the 'Yes and No' point. In such a case, it is difficult to understand immediately how many logo trend models are located at each point.

Based on the pros and cons of the two aforementioned visualization methods, we concluded that RadViz visualization requires an auxiliary means to support intuitively viewing the shape elements of each logo trend model in detail. We also concluded that parallel coordinates fulfill this requirement. Accordingly, we also concluded that using these two visualization methods simultaneously would provide suitable results in displaying logo trend visualizations.

However, directly implementing the linear form of parallel coordinates without modifications would cause gaze dispersion when the user switches between both visualizations. Furthermore, even when applying a large number of variables, there exists a problem of poor readability because the axes must be compactly placed in a linear space.

Thus, through assessments with the experts, we tried to devise a harmonious way to integrate both visualization techniques. The combination of RadViz and circular parallel coordinates was selected as the final model system for visualization. This visualization is intended to secure readability in understanding the data characteristics through presentation of the data variables by matching the positions of the dimensional anchors of the RadViz and the axes of the parallel coordinates. The process of implementing this visualization and its results are described in detail in the next section.

\section{The Logo Trend Visualization System}

This section introduces logo trend visualization based on design shape element data of logos that have been quantified earlier. Our visualization system is in a circular form with parallel coordinates around the ring where the dimensional anchors of RadViz are represented.

The reason for combining two visualization techniques (RadViz and the circular parallel coordinates) in this study is that both serve different purposes. RadViz helps in representing the cluster of logo trend model while the circular parallel coordinates allow us to analyze the details of logo design elements. 
Therefore, in order to solve this problem, this study incorporates the parallel coordinates in a circular form along with RadViz. The nodes within the RadViz visualization are specified to be powered by the shape element dimensional anchor of each trend.

Moreover, in order to cluster logo trend nodes with similar characteristics, weights are given according to the type of basic elements (concept element, visual element, correlation element, and the interrelationships of form). The equation for specifying the position value equation $P$ and the weight $W$ of the node is as follows:

$$
P=\frac{\sum_{c=0}^{c n} \sum_{n=1}^{k(c)} f p(c, n)+\sum_{n=1}^{k(f)}(f p(f, n) \times W)}{\sum_{c=0}^{c n} k(c)+W \times k(f)}, W=\sum k(f)
$$

where $f p(c, n)$ represents the position value of the element that affects the node located at $n$ of the basic shape element $c$, and $k(c)$ returns the elements that affect the basic shape element of the node. In addition, $c n$ refers to the number of basic shape elements and $f$ represents the basic shape element in which the node has a weight. The weight, $W$, can be represented by the equation on the right, where $k(f)$ returns the elements that affect the basic shape element of the node. The weighted value of the weight is the sum of the constant values that affect the node within the basic shape element.

This study has implemented the sample visualization model through the above process, and the final logo trend visualization map is shown in Figure 10.

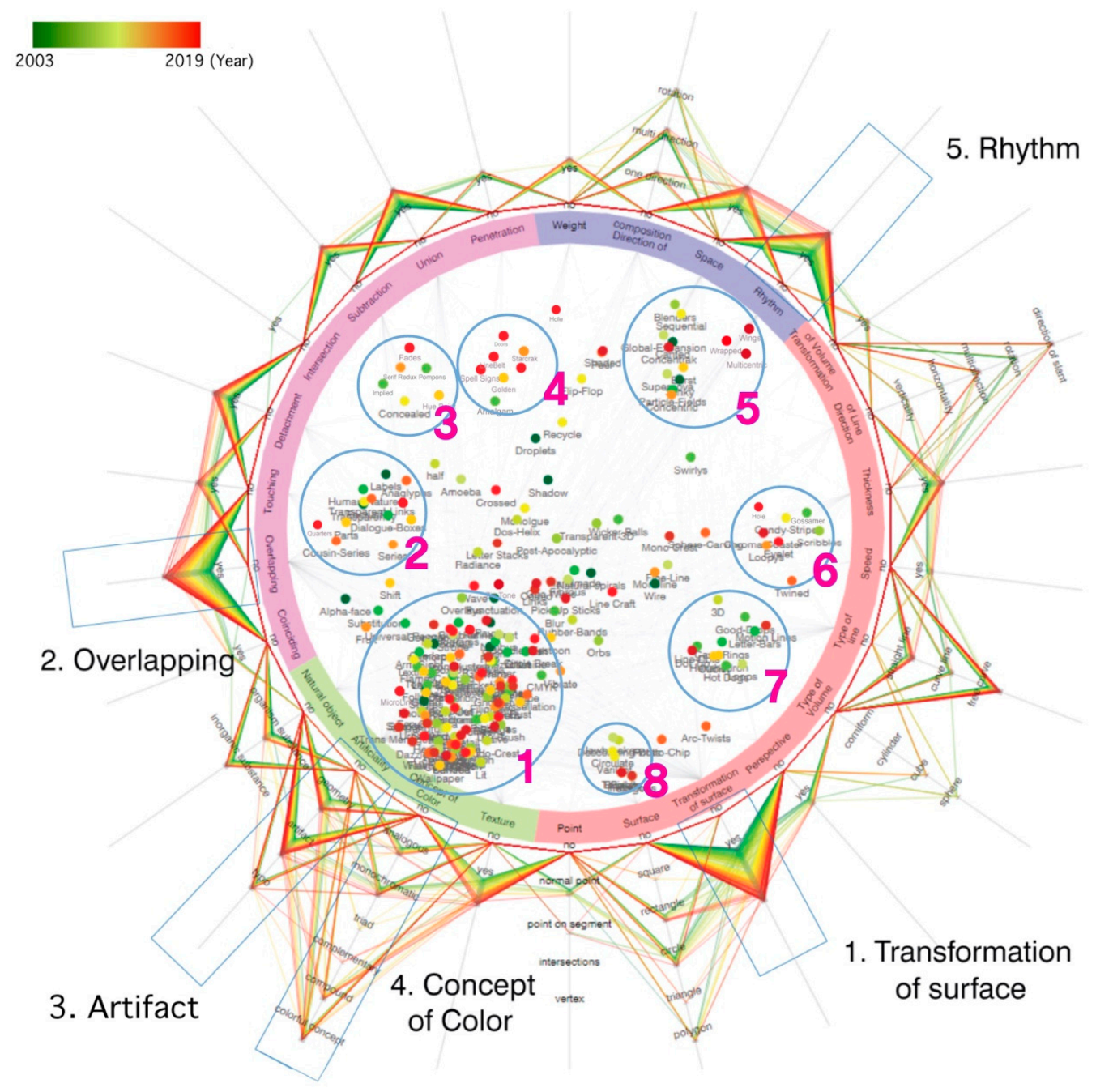

Figure 10. Final logo trend map. Updated from Reference [10]; 2015. 
The color of the circular parallel coordinates indicates the line pattern of a more recent year as it moves from the green to a red line pattern. Each node in the RadViz plot represents one logo trend. There are also keywords representing the shape elements on the four colored ring-shaped bands around the circle. Each point where this keyword appears becomes a dimensional anchor that pulls the nodes. Therefore, the logo nodes are powered by the dimensional anchors indicated by the keyword, and they form a scatter plot. The nodes in close proximity to each other indicate trends with similar design shape elements.

Each line of the circular parallel coordinates plot presents the detailed shape element of one logo trend node. Therefore, by following the line graphs of the circle parallel coordinates, the main shape elements of a logo trend can be seen at a glance.

Looking at the nodes distributed in the RadViz plot, most of them are driven by the force around the circle where the keywords 'Transformation of Surface, Overlapping, Artifact, and Concept of Color' are located. Such a result indicates that it is desirable to transform the surface with the forms overlapping using artificial visual elements, while giving a sense of rhythm in the use of various colors and the arrangement of shapes for a logo to reflect a trend. Therefore, it can be concluded that the concept element, visual element, correlation element, and the interrelationships of forms should be evenly combined for a logo to reflect a trend.

With the RadViz visualization of this study, we found that logo trend models with similar design shape elements cluster together. Figure 11 shows eight cluster patterns and corresponding logo trend models that can be found on RadViz visualization in Figure 10. Table 2 shows the summary of the design shape elements used by logo trends included in the eight clusters. The elements commonly used in each cluster are displayed in bold.
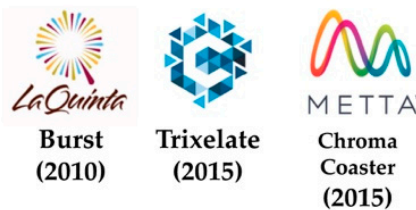

Cluster 1: Natural or Artificial Object, Colorful and Compound concept
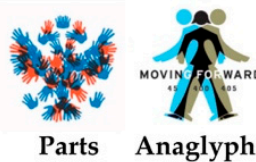

(2010)

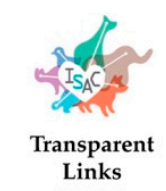

(2012)

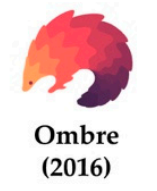

2016)

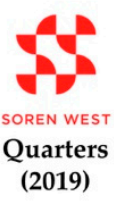

Cluster 2: Overlapping and Touching
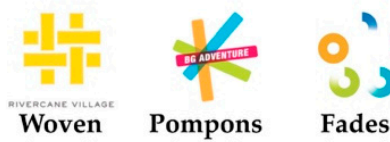

(2014)

LeIN IKU BOTÍN rENTDE

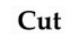

(2018)

Cluster 3: Subtraction and Intersection
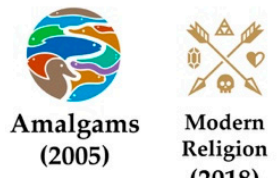
(2018)
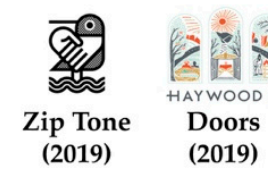

Cluster 4: Union of various objects
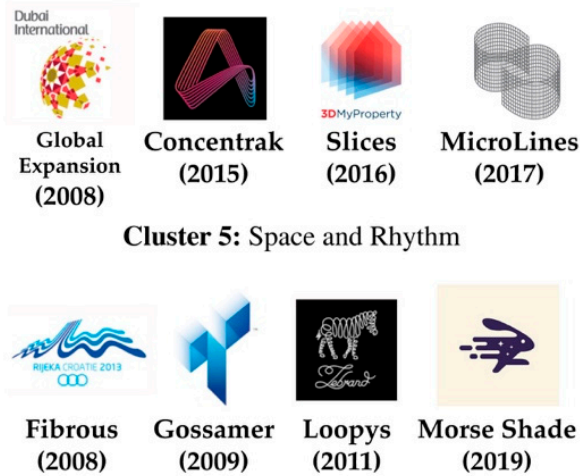

Cluster 6: Speed

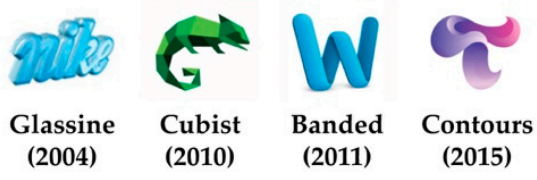

Cluster 7: Volume concept (3D)

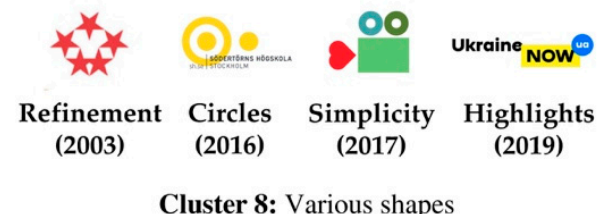

Cluster 8: Various shapes

Figure 11. Eight clusters along with design shape element patterns and logo trend samples. Reproduced by using Reference [4]; 2020. 
Table 2. Details of the eight clusters based on design shape elements. (The elements commonly used in each cluster are displayed in bold.)

\begin{tabular}{|c|c|c|c|}
\hline Cluster & Design Shape Elements & Cluster & Design Shape Elements \\
\hline 1 & $\begin{array}{l}\text { Natural Object (Organism } \\
\text { substance), Artificiality (Geometry, } \\
\text { Artifact), Concept of Color } \\
\text { (Monochromatic, Compound, } \\
\text { Colorful concept), Union }\end{array}$ & 5 & $\begin{array}{l}\text { Surface (Transformation of } \\
\text { surface, Perspective), Volume } \\
\text { (Transformation of volume), } \\
\text { Artificiality (Geometry), } \\
\text { Rhythm, Texture, Space }\end{array}$ \\
\hline 2 & $\begin{array}{l}\text { Overlapping, Touching, Concept of } \\
\text { Color (Compound, Triad), } \\
\text { Intersection, Composition } \\
\text { (Multi-direction) }\end{array}$ & 6 & $\begin{array}{c}\text { Speed, Rhythm, Overlapping, } \\
\text { Artificiality (Artifact, Geometry), } \\
\text { Monochromatic }\end{array}$ \\
\hline 3 & $\begin{array}{c}\text { Subtraction, Intersection, } \\
\text { Artificiality (Typography), Surface } \\
\text { (Rectangle), Line (Straight Line), } \\
\text { Thickness, Verticality }\end{array}$ & 7 & $\begin{array}{c}\text { Volume (Cube, Transformation } \\
\text { of volume), Polygon, } \\
\text { Artificiality (Artifact, Geometry) }\end{array}$ \\
\hline 4 & $\begin{array}{c}\text { Union, Touching, Natural Object } \\
\text { (Organism Substance), Artificiality } \\
\text { (Artifact), Texture, Space }\end{array}$ & 8 & $\begin{array}{l}\text { Various Concept Element } \\
\text { (Point, line, Surface), Union, } \\
\text { Touching, Overlapping, } \\
\text { Artificiality (Typography) }\end{array}$ \\
\hline
\end{tabular}

First of all, cluster 1 is composed with natural or artificial objects whose color concept is colorful or compound, and trends of objects that are either overlapping or touching can be seen at cluster 2 . Trends with parts of an object either subtracting or intersecting each other are grouped at cluster 3 . At cluster 4, trends with various objects united together can be seen. At cluster 5, trends with space and rhythm are congregated, and at cluster 6, trends with speed are gathered together. Cluster 7 is made out of trends with three-dimensional (3D) shape. Lastly, trends that have a particular surface, such as triangle, rectangle, or circle, as a concept, are congregated at cluster 8. Furthermore, as shown in Table 2, each cluster has its own distinctive shape elements in addition to a wide variety of frequently used shape elements, namely 'Transformation of Surface, Overlapping, Artifact, and Concept of Color'.

\section{Case Studies to Identify the Trend of Logos}

\subsection{Case 1: A Report on the Evolution of Design Shape Element Patterns}

The visual analysis presented in this study cannot only help understand the evolution of logo trends but also predict the trend of logos. To support this, this section presents a case study to identify the evolution of a logo trend.

First, the visualization system described in Section 5 was used to investigate the design shape elements frequently used by year. Next, the color of the logo trend node expressed in RadViz and the shape elements of the logo trend according to the released year were observed. After analyzing the detailed shape elements of each logo by looking at the dimensional anchor and the circular parallel coordinates plot, the frequently used shape elements were summarized in Table 3.

Table 3 shows the evolution of trends from 2003 to 2019 as analyzed through the logo trend visualization system. It can be seen that various changes in the shape elements occurred from 2003 to 2012, and the shape elements that were popular in 2003-2006 reappeared during 2013-2015. From 2016, as simple logos became a new trend, shape elements such as 'Monochromatic' and 'Typography' began trending. In 2017, shape elements closely related to line elements were popular, and the expression of concepts such as change in line thickness, rhythm, space, and geometry became the mainstream. In 2018 and 2019, due to the emergence of a shape element called subtraction, which had never been seen before, a new trend was introduced with the appearance of logos being partially cut off or omitted. Like this, using the visualization system of this study, it is possible to observe the changes in the trends of frequently used shape elements and logos by year. 
Table 3. Flow of logo trends from 2003 to 2019.

\begin{tabular}{|c|c|c|c|}
\hline Year & Frequently Used Shape Elements & Year & Frequently Used Shape Elements \\
\hline 2003 & $\begin{array}{l}\text { Transformation of Surface, Free Curve, } \\
\text { Polygon, Artifact, Overlapping }\end{array}$ & 2012 & $\begin{array}{l}\text { Union, Geometry, Artifact, } \\
\text { Transformation of surface, Space, Square, } \\
\text { Rectangle }\end{array}$ \\
\hline 2004 & $\begin{array}{l}\text { Detachment, Geometry, Transformation } \\
\text { of Surface, Point, Circle, Multi-direction }\end{array}$ & 2013 & $\begin{array}{c}\text { Transformation of surface, Free Curve, } \\
\text { Volume, Artifact, Overlapping, } \\
\text { Analogous }\end{array}$ \\
\hline 2005 & $\begin{array}{l}\text { Space, Rhythm, Union, Transformation } \\
\text { of surface, Free curve }\end{array}$ & 2014 & $\begin{array}{l}\text { Space, Union, Rhythm, Transformation of } \\
\text { surface, Free curve }\end{array}$ \\
\hline 2006 & $\begin{array}{l}\text { Volume, Rhythm, Speed, Overlapping, } \\
\text { Colorful concept }\end{array}$ & 2015 & $\begin{array}{c}\text { Geometry, Natural object, Colorful } \\
\text { Concept, Transformation of Surface, } \\
\text { Triangle }\end{array}$ \\
\hline 2007 & $\begin{array}{l}\text { Colorful concept, Volume, Rhythm, } \\
\text { Geometry, Natural object }\end{array}$ & 2016 & $\begin{array}{l}\text { Transformation of Surface, Polygon, } \\
\text { Volume, Typography, Monochromatic, } \\
\text { Analogous, Overlapping, Union }\end{array}$ \\
\hline 2008 & $\begin{array}{l}\text { Natural object, Transformation of } \\
\text { surface, Speed, Rhythm, Detachment }\end{array}$ & 2017 & $\begin{array}{l}\text { Free curve, Colorful concept, Geometry, } \\
\text { Texture, Rhythm, Space, Weight, } \\
\text { Thickness, Multi direction, Detachment, } \\
\text { Overlapping }\end{array}$ \\
\hline 2009 & $\begin{array}{l}\text { Colorful concept, Overlapping, } \\
\text { Transformation of surface, Polygon }\end{array}$ & 2018 & $\begin{array}{c}\text { Texture, Rhythm, Typography, Thickness, } \\
\text { Transformation of surface, } \\
\text { Monochromatic, Analogous, } \\
\text { Complementary, Subtraction }\end{array}$ \\
\hline 2010 & $\begin{array}{l}\text { Volume, Rhythm, Speed, Space, } \\
\text { Transformation of surface, Overlapping }\end{array}$ & 2019 & $\begin{array}{l}\text { Geometry, Speed, Transformation of } \\
\text { surface, Rhythm, Union, Subtraction }\end{array}$ \\
\hline 2011 & $\begin{array}{l}\text { Analogous, Natural object, Rhythm, } \\
\text { Space, Detachment }\end{array}$ & & \\
\hline
\end{tabular}

\subsection{Case 2: Logo Trends That Evolve across Different Clusters in the RadViz}

Figures 12 and 13 show how the logo trend "Wire" in 2004 evolved over time into a new trend.

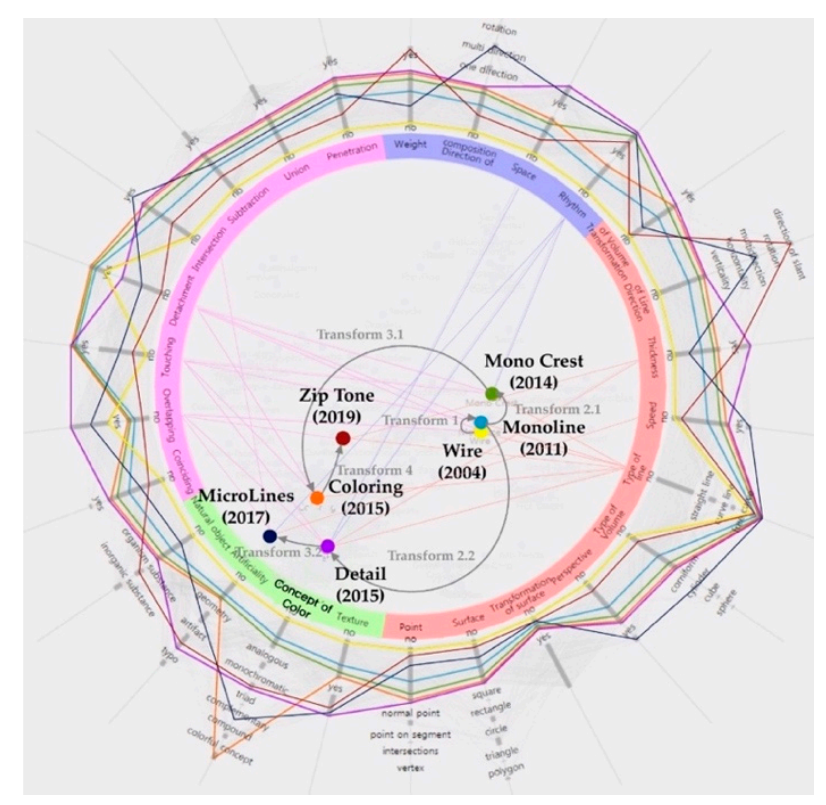

Figure 12. RadViz and circle parallel coordinates showing how the 2004 logo trend "Wire" was transformed into a new trend over time. Adapted from Reference [10]; 2015. 


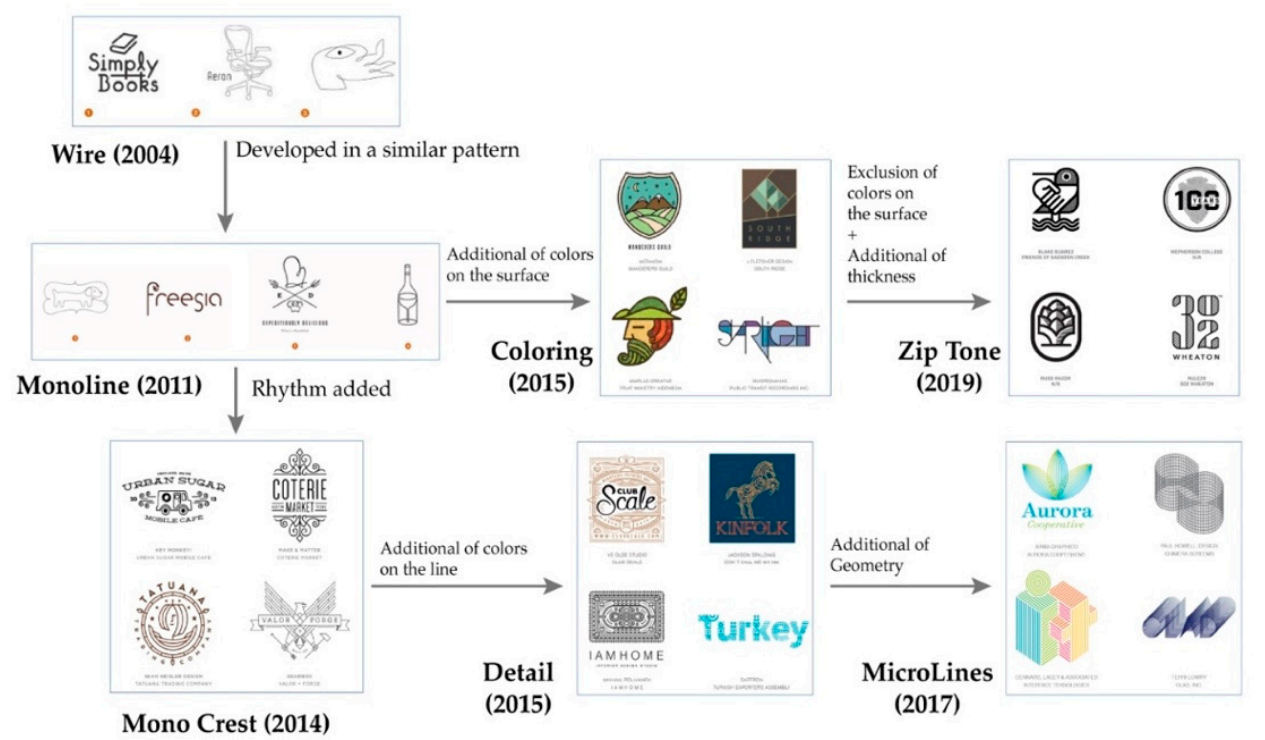

Figure 13. Evolution of the logo trend, "Wire". Updated from Reference [10]; 2015.

First, it can be seen that the node "Wire" (yellow) and the node "Monoline" (blue) are located close to each other. The shapes of their circular parallel coordinates (yellow and blue line graphs) are almost similar as well.

In fact, the article in the annual Logo Trend Report stated that the "Wire" trend had appeared in 2004 and the "Monoline" trend came out seven years later, in 2011, which was largely in keeping with the "Wire" trend. Given this fact, it can be seen that if the nodes have similar characteristics, they are in close proximity to each other.

Next, the trends "Coloring" (orange) and "Mono Crest" (green) are somewhat far apart from each other. The node "Coloring" (orange) is shown to be located near the 'Concept of Color' dimensional anchor of shape elements. In addition, when looking at the circular parallel coordinates plot, the line graph expressing the "Coloring" trend is markedly different from the line graph expressing the "Mono Crest" trend in the axis portion of 'Concept of Color.' In fact, the 2015 Logo Trend Report confirmed that the "Coloring" trend was created by adding 'Colors on the Surface' based on the "Mono Crest" trend of 2014. Given this, it can be seen that new trends can be created due to changes in the same basic element area and that they can also be created by adding shape elements in other basic element areas. In other words, the "Mono Crest" trend based on "Wire" is a new logo trend created due to changes in "line," which is a concept element.

On the other hand, "Coloring" is a new logo trend created by the addition of a shape element, 'Concept of Color', which is a visual element and not a concept element. Therefore, it can be seen that new trends are created when different basic shape elements are changed as well. Also, the "Mono Crest" trend evolved into the "Detail" (purple) trend in 2015, with the addition of the concept of "Colors on the Line.' Four years later, the "Coloring" trend led to the "Zip Tone" (dark red) trend. Later, the previously added 'Concept of Color' was removed again and the concept of changing the thickness of the line was added.

The "Detail" trend of 2015, inspired by hand-painted illustrations, led to the "MicroLines" (navy) trend in 2017, which is, in fact, conceptually the opposite of the "Detail" trend. It can be seen that the design was transformed into a more geometric design utilizing space and three-dimensional impression. Additionally, we discovered more evolution patterns in the process of adding logo trend cases and observing the cluster characteristics displayed in the visualization system. 


\subsection{Case 3: Logo Trends Evolving within the Same Cluster}

First, the patterns of evolution were observed when we analyzed the visualization of logo trends present in a specific cluster. Figure 14 shows how the logo trend "Mosaic" in 2009 evolved over time into a new trend. The trends that belong to the "Mosaic" trend evolution fall under Cluster 1 in RadViz as these trends feature combinations of objects having different colors. In the "Mosaic" trend, the shape element characteristics are unions of surfaces having colorful concepts.

In 2010, the trend evolved to "Pixels", which had similar color concepts and surfaces to the previous trend. One difference of the "Pixels" trend is that it is composed of objects with square- and rectangle-shaped surfaces to match the name "Pixels".

Based on the "Pixels" trend, similar trends appeared in 2011 and 2012 after implementing different shape elements. The "Bucky" trend in 2011 featured triangular surfaces that were different from the design patterns of the "Pixels" trend. Furthermore, in the "Bucky" trend, the characteristic of line thickness was added to make each object seem separated from each other. For the "Tessellation" trend in 2012, various types of surfaces were coordinated, and various elements related to natural objects such as butterflies and fish were added to display the logo transformation.

The trends described above became integrated and evolved to the "Trixelate" trend in 2015. Although this trend had similar surface characteristic to the "Bucky" trend, the existing colorful concept was removed, acquiring a monochromatic and analogous color concept. Additionally, in contrast with the "Tessellation" trend, the natural object element was removed and more artificial shape elements ('Artifact' and 'Geometry') were incorporated in the "Trixelate" trend.

Figure 15 shows how the logo trend "Concentric", created in 2011, evolved over time into a new trend. The trends belonging to the evolution of "Concentric" fall under Cluster 5 in RadViz as they share elements that commonly show rhythm. The "Concentric" trend has the characteristic of conveying a feeling of rhythm in a process where numerous lines are arranged into a repetitive pattern. After 2011, this trend received attention again in 2015 in relation with a trend called "Concentrak", with similar shape elements. Then, the trend further evolved to "MultiCentric" in 2017. As shown from this trend evolution, the line thickness changed and the monochromatic color concept, which was maintained in the previous trends, evolved to a colorful concept in the latest trend.

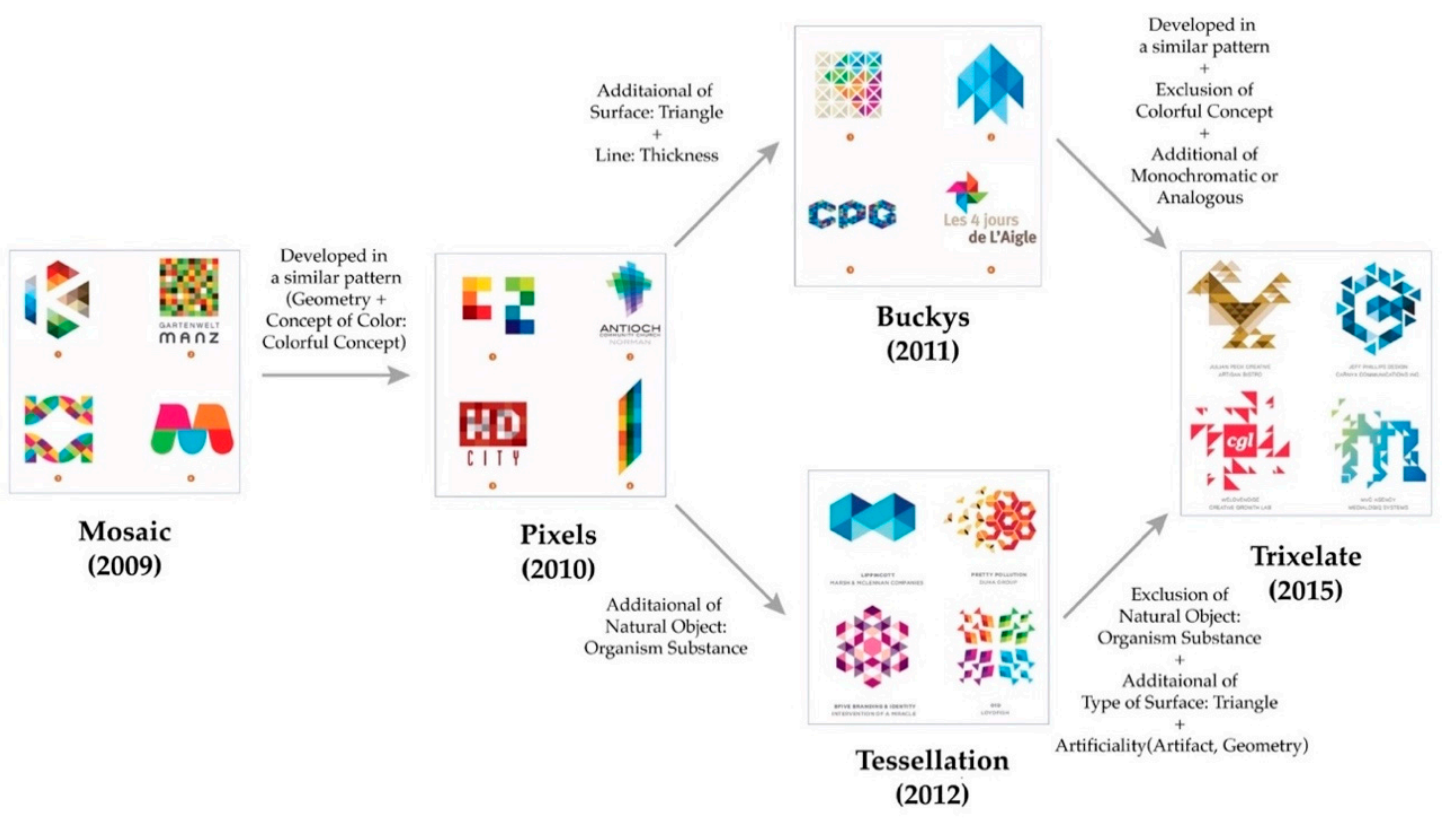

Figure 14. Evolution of the logo trend, "Mosaic". 


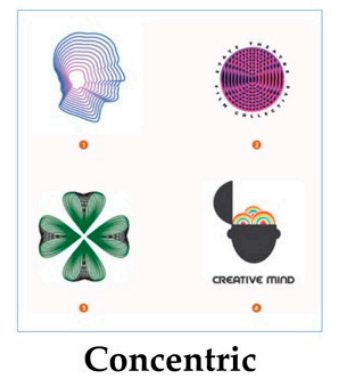

(2011)

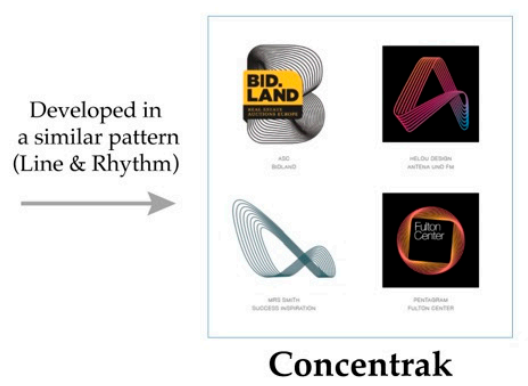

(2015)
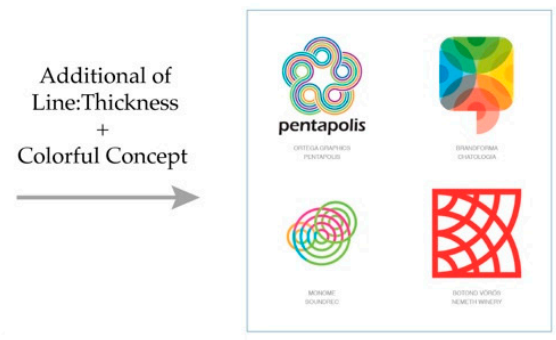

MultiCentric

(2017)

Figure 15. Evolution of the logo trend, "Concentric".

Based on the above case study, we have been able to effectively show how the shape elements of a specific logo trend can change over time. The results of the visualization trend analysis of this study can be used to supplement the classification system of logos and to produce logos reflecting new trends.

\section{Conclusions}

This study prepared the classification criteria based on the Principles of Form and Design for logo classification and quantified the logo trend data accordingly. RadViz and circular parallel coordinates visualization methods were used to implement the logo trend map based on the quantified data. We were able to identify the frequently used shape elements through the visualization system that was created. In addition, we were also able to understand how the concept of a shape element changes when a logo trend leads to a new trend over time. In particular, eight cluster patterns featuring similar shape elements were extracted after applying a large number of logo trend resources. In each cluster, unique design shape elements were incorporated in addition to elements frequently used in the data of this study. This can be considered as a successful result in contrast with our previous work, where logo cluster trends could not be clearly identified and separated. Additionally, the evolutions of logo trend patterns were observed in both cases, i.e., analysis of one cluster and analysis of multiple clusters simultaneously.

Our research helps in comparing and judging trends that fit the actual situation because it provides a classification system for aesthetic, geometric, and formative elements, while reflecting the latest logo trend information. Recently, many companies have succeeded in changing their corporate image by producing logos that are both professional and current. Using our system as a guideline, many companies would be able to produce logos that reflect what is trending.

However, whether the general public or beginners in graphic design who intend to create logos are able to use our analysis tools to understand the trend of logo trends requires further confirmation since the system in our study has been created targeting those who are engaged in logo design or those evaluating design trends.

Therefore, in the future, we plan to verify the effectiveness of this study after conducting usability verification experiments on general users in all fields who intend to make or analyze logos. In addition, we intend to improve the proposed model further by examining inconveniences of the proposed visualization method and enhancing the technical elements.

Author Contributions: Conceptualization, H.H. (Hyoji Ha) and K.L.; Methodology, H.H. (Hyoji Ha) and K.L.; Software, H.H. (Hyunwoo Han); Validation, H.H. (Hyoji Ha), H.H. (Hyunwoo Han) and K.L.; Formal Analysis, H.H. (Hyoji Ha), H.H. (Hyunwoo Han) and K.L.; Investigation, H.H. (Hyoji Ha); Data Curation, H.H. (Hyoji Ha) and H.H. (Hyunwoo Han); Writing-Original Draft Preparation, H.H. (Hyoji Ha); resources, H.H. (Hyoji Ha) and H.H. (Hyunwoo Han); Writing-Review and Editing, H.H. (Hyoji Ha), H.H. (Hyunwoo Han) and K.L.; Visualization, H.H. (Hyoji Ha), H.H. (Hyunwoo Han) and K.L.; Supervision, K.L. All authors have read and agreed to the published version of the manuscript. 
Funding: This research was funded by The Ministry of Education of the Republic of Korea and the National Research Foundation of Korea, grant number (NRF-2018S1A5B6075104), and Brain Korea 21 Plus Digital Therapy Research Team, grant number (NRF31Z20130012946).

Conflicts of Interest: The authors declare no conflict of interest.

\section{References}

1. Kim, H.K.; Kim, Y.I. The Analysis of the Influential Factors on Design Trends and Color Trends in the Late 20th Century. Arch. Des. Res. 2007, 20, 5-20.

2. Lee, H.E. A Study on the Process Model for "Design Trend Forecasting"—Focusing on Designer's Experiences by Using Their Intuition. J. Korean Soc. Des. Cult. 2014, 20, 499-510.

3. Airey, D. Logo Design Love: A Guide to Creating Iconic Brand Identities; New Riders: Indianapolis, IN, USA, 2009.

4. Shih, A.C.C.; Lee, D.T.; Peng, C.L.; Wu, Y.W. Phylo-mLogo: An interactive and hierarchical multiple-logo visualization tool for alignment of many sequences. BMC Bioinform. 2007, 8, 63. [CrossRef]

5. Johari, M.; Azmeer, R.A.; Dolah, M.S. Development of the Recognisable Logo Design Guideline for Startup Brands. Int. J. Sci. Res. Manag. 2019, 7. [CrossRef]

6. Annual Logo Trends. Available online: https://www.logolounge.com/trend-reports (accessed on 20 May 2020).

7. Luffarelli, J.; Stamatogiannakis, A.; Yang, H. The visual asymmetry effect: An interplay of logo design and brand personality on brand equity. J. Mark. Res. 2019, 56, 89-103. [CrossRef]

8. Bossel, V.; Geyskens, K.; Goukens, C. Facing a trend of brand logo simplicity: The impact of brand logo design on consumption. Food Qual. Prefer. 2019, 71, 129-135. [CrossRef]

9. Van Grinsven, B.; Das, E. Logo design in marketing communications: Brand logo complexity moderates exposure effects on brand recognition and brand attitude. J. Mark. Commun. 2016, 22, 256-270. [CrossRef]

10. Ha, H.; Bae, S.; Han, H.; Lee, K. Logo Trend Visualization: Logo Design Shape Element Trend and Pattern Analysis. In Proceedings of the 8th International Symposium on Visual Information Communication and Interaction, Tokyo, Japan, 24-26 August 2015; pp. 158-159.

11. Neumann, J.; Samet, H.; Soffer, A. Integration of local and global shape analysis for logo classification. Pattern Recognit. Lett. 2002, 23, 1449-1457. [CrossRef]

12. Trademark \& Design Examination Bureau. Vienna Classification, 6th ed.; Korean Intellectual Property Office: Daejeon, Korea, 2010; pp. 221-245.

13. Kang, K.M.; Kim, C.K.; Lee, D. A Study on Similarity of Logo-Image for Protecting Illegal Usage. In Proceedings of the Korean Institute of Information Scientists and Engineers, Jeju, Korea, 30 June-2 July 2010; pp. 357-361.

14. Wei, C.H.; Li, Y.; Chau, W.Y.; Li, C.T. Trademark image retrieval using synthetic features for describing global shape and interior structure. Pattern Recognit. 2009, 42, 386-394. [CrossRef]

15. Qi, H.; Li, K.; Shen, Y.; Qu, W. An effective solution for trademark image retrieval by combining shape description and feature matching. Pattern Recognit. 2010, 43, 2017-2027. [CrossRef]

16. Zhang, J.; Yu, J.; Zhang, K.; Zheng, X.S.; Zhang, J. Computational aesthetic evaluation of logos. ACM Trans. Appl. Percept. 2017, 14, 20. [CrossRef]

17. Fayyad, U.M.; Wierse, A.; Grinstein, G.G. Information Visualization in Data Mining and Knowledge Discovery; Morgan Kaufmann: Burlington, MA, USA, 2002.

18. Ha, H.; Han, H.; Mun, S.; Bae, S.; Lee, J.H.; Lee, K. An Improved Study of Multilevel Semantic Network Visualization for Analyzing Sentiment Word of Movie Review Data. Appl. Sci. 2019, 9, 2419. [CrossRef]

19. Ghani, S.; Kwon, B.C.; Lee, S.; Yi, J.S.; Elmqvist, N. Visual analytics for multimodal social network analysis: A design study with social scientists. IEEE Trans. Vis. Comput. Gr. 2013, 19, 2032-2041. [CrossRef] [PubMed]

20. Beham, M.; Herzner, W.; Gröller, M.E.; Kehrer, J. Cupid: Cluster-based exploration of geometry generators with parallel coordinates and radial trees. IEEE Trans. Vis. Comput. Gr. 2014, 20, 1693-1702. [CrossRef] [PubMed]

21. Ismail, A.; Shehab, A.; El-Henawy, I.M. Healthcare Analysis in Smart Big Data Analytics: Reviews, Challenges and Recommendations, 1st ed.; Springer Nature Switzerland AG: Basel, Switzerland, 2019; pp. 27-45.

22. Husnain, M.; Missen, M.M.S.; Mumtaz, S.; Luqman, M.M.; Coustaty, M.; Ogier, J.M. Visualization of High-Dimensional Data by Pairwise Fusion Matrices Using t-SNE. Symmetry 2019, 11, 107. [CrossRef]

23. Wang, J.; Liu, X.; Shen, H.W. High-dimensional data analysis with subspace comparison using matrix visualization. Inf. Vis. 2019, 18, 94-109. [CrossRef] 
24. Tong, C.; Zhang, J.; Chowdhury, A.; Trost, S.G. An Interactive Visualization Tool for Sensor-based Physical Activity Data Analysis. In Proceedings of the Australasian Computer Science Week Multiconference, Sydney, NSW, Australia, 29-31 January 2019; p. 30.

25. Hoffman, P.; Grinstein, G.; Marx, K.; Grosse, I.; Stanley, E. DNA visual and analytic data mining. In Proceedings of the Visualization'97 (Cat. No. 97CB36155), Phoenix, AZ, USA, 19-24 October 1997; pp. 437-441.

26. Grinstein, G.; Jessee, C.B.; Hoffman, P.; O’Neil, P.; Gee, A.; Grigorenko, E.V. High-dimensional visualization support for data mining gene expression data. DNA Arrays Technol. Exp. Strateg. 2001, 86-131.

27. Daniels, K.; Grinstein, G.; Russell, A.; Glidden, M. Properties of normalized radial visualizations. Inf. Vis. 2012, 11, 273-300. [CrossRef]

28. Sharko, J.; Grinstein, G.; Marx, K.A. Vectorized radviz and its application to multiple cluster datasets. IEEE Trans. Vis. Comput. Gr. 2008, 14, 1444-1451. [CrossRef]

29. De Carvalho Pagliosa, L.; Telea, A.C. RadViz: Improvements on Radial-Based Visualizations++. Informatics 2019, 6, 16. [CrossRef]

30. Zhou, F.; Huang, W.; Li, J.; Huang, Y.; Shi, Y.; Zhao, Y. Extending Dimensions in Radviz based on mean shift. In Proceedings of the 2015 IEEE Pacific Visualization Symposium, Hangzhou, China, 14-17 April 2015; pp. 111-115.

31. Inselberg, A. The plane with parallel coordinates. Vis. Comput. 1985, 1, 69-91. [CrossRef]

32. Inselberg, A.; Dimsdale, B. Parallel coordinates for visualizing multi-dimensional geometry. In Computer Graphics; Springer: Tokyo, Japan, 1987; pp. 25-44.

33. Inselberg, A. Parallel Coordinates: Visual Multidimensional Geometry and Its Applications; Springer: Berlin/Heidelberg, Germany, 2009.

34. Wegman, E.J.; Luo, Q. High dimensional clustering using parallel coordinates and the grand tour. In Classification and Knowledge Organization; Springer: Berlin/Heidelberg, Germany, 1997; pp. 93-101.

35. Johansson, J.; Forsell, C. Evaluation of parallel coordinates: Overview, categorization and guidelines for future research. IEEE Trans. Vis. Comput. Gr. 2015, 22, 579-588. [CrossRef] [PubMed]

36. Zhou, H.; Yuan, X.; Qu, H.; Cui, W.; Chen, B. Visual clustering in Parallel Coordinates. Comput. Gr. Forum 2008, 27, 1047-1054. [CrossRef]

37. Raseman, W.J.; Jacobson, J.; Kasprzyk, J.R. Parasol: An open source, interactive parallel coordinates library for multi-objective decision making. Environ. Model. Softw. 2019, 116, 153-163. [CrossRef]

38. Cheng, S.; Mueller, K. Improving the fidelity of contextual data layouts using a generalized barycentric coordinates framework. In Proceedings of the 2015 IEEE Pacific Visualization Symposium, Hangzhou, China, 14-17 April 2015; pp. 295-302.

39. Gardner, B.; Fishel, C. LogoLounge 5: 2000 International Identities by Leading Designers; Rockport Publishers: London, UK, 2011; pp. 84-180.

40. Wong, W. Principles of Form and Design, 1st ed.; John Wiley \& Sons: Hoboken, NJ, USA, 1993; pp. 41-49.

41. Wong, W. Principles of Color Design, 1st ed.; John Wiley \& Sons: Hoboken, NJ, USA, 1997; pp. $25-55$.

42. Wong, W. Principles of 2-Dimensional Design, 1st ed.; John Wiley \& Sons: Hoboken, NJ, USA, 1972; pp. 5-14.

43. Ono, J.H.P.; Sikansi, F.; Corrêa, D.C.; Paulovich, F.V.; Paiva, A.; Nonato, L.G. Concentric radviz: Visual exploration of multi-task classification. In Proceedings of the 2015 28th SIBGRAPI Conference on Graphics, Patterns and Images), Salvador, Brazil, 26-29 August 2015; pp. 165-172.

44. Hoffman, P.; Grinstein, G.; Pinkney, D. Dimensional anchors: A graphic primitive for multidimensional multivariate information visualizations. In Proceedings of the 1999 Workshop on New Paradigms in Information Visualization and Manipulation (NPIVM '99), in conjunction with the Eighth ACM International Conference on Information and Knowledge Management (CIKM '99), Kansas City, MO, USA, 6 November 1999; pp. 9-16.

45. Ha, H.; Lee, J.; Han, H.; Bae, S.; Son, S.; Hong, C.; Shin, H.; Lee, K. Dementia Patient Segmentation Using EMR Data Visualization: A Design Study. Int. J. Environ. Res. Public Health 2019, 16, 3438. [CrossRef] [PubMed] 